The Open Statistics \& Probability
Journal
CrossMark
Content list available at: www.benthamopen.com/TOSPJ/
DOI $10.2174 / 1876527001809010026,2018,9,26-41$

RESEARCH ARTICLE

\title{
Asymptotic Relative Efficiencies of the Score and Robust Tests in Genetic Association Studies
}

\author{
Ao Yuan ${ }^{1, *}$, Ruzong Fan ${ }^{1}$, Jinfeng $\mathrm{Xu}^{2}$, Yuan Xue ${ }^{3}$ and Qizhai $\mathrm{Li}^{4}$ \\ ${ }^{I}$ Department of Biostatistics, Bioinformatics and Biomathematics, Georgetown University, Washington DC, 20057, \\ USA \\ ${ }^{2}$ Department of Statistics and Actuarial Science, University of Hong Kong, Hong Kong \\ ${ }^{3}$ School of Mathematical Sciences, University of Chinese Academy of Sciences, Beijing, 100190, China \\ ${ }^{4}$ LSC, NCMIS, Academy of Mathematics and Systems Science, Chinese Academy of Sciences, Beijing, 100190, China
}

Received: March 7, 2018

Revised: July 23, 2018

Accepted: October 2, 2018

\begin{abstract}
:
Introduction:

The score statistic $Z(\theta)$ and the maximin efficient robust test statistic $Z_{M E R T}$ are commonly used in genetic association study, but according to our knowledge there is no formal comparison of them.

\section{Methods:}

In this report, we compare the asymptotic behavior of $Z(\theta)$ and $Z_{M E R T}$, by computing their Asymptotic Relative Efficiencies (AREs) relative to each other. Four commonly used ARE measures, the Pitman ARE, Chernoff ARE, Hodges-Lehmann ARE and the Bahadur ARE are considered. Some modifications of these methods are made to simplify the computations. We found that the Chernoff, Hodges-Lehmann and Bahadur AREs are suitable for our setting.
\end{abstract}

\section{Results and Conclusion:}

Based on our study, the efficiencies of the two test statistic varies for different criterion used, and for different parameter values under the same criterion, so each test has its advantages and dis-advantages according to the criterion used and the parameters involved, which are described in the context. Numerical examples are given to illustrate the use of the two statistics in genetic association study.

Keywords: Asymptotic relative efficiency, Genetic association study, Maximin efficiency robust test, Score test $Z(\theta)$, Test statistic $\mathrm{Z}_{\text {mert }}$, Pitman ARE, Chernoff ARE.

\section{INTRODUCTION}

In genetic association studies, several test statistics are often used, including the score test $Z(\theta)$ and the maximin efficient robust test statistic $Z_{M E R T}$. Although numerical behavior of the two tests are reported in various genetic association studies based on simulations, to our knowledge, a formal theoretical comparison of the two tests hasn't been seen in the literature. It is of meaning to compare their asymptotic performances. Although for likelihood ratio based test statistic for testing hypothesis of simple null versus simple alternative, there is a uniformly most powerful test under some regularity conditions. However, most test statistics are not constructed directly from likelihood ratio, the hypothesis are composite, and there is generally no such optimal test. Therefore, the classical method to compare any two test statistics is to evaluate the Asymptotic Relative Efficiency (ARE) between them.

\footnotetext{
* Address correspondence to this author at the Department of Biostatistics, Bioinformatics and Biomathematics, Georgetown University, Washington DC, 20057, USA, Tel: +91 22 33611111, E-mail: ay312@georgetown.edu
} 
The ARE is a well studied area, with vast literatures and numerous different definitions. But often the computation of ARE is very difficult in the general case, some of the classical methods for ARE require that the test statistics have some standard forms, such as they have the same asymptotic distribution, or have the forms of i.i.d. summations. However, in practice, such as in genetic association studies, some test statistics do not have these forms. Sitlani and McKnight [1] studied AREs for the trend test under different models and stratifications. In this communication, wecompare the asymptotic behavior of two commonly used test statistics the score statistic $Z(\theta)$ and the maximin efficient robust test statistic $Z_{M E R T}$, arise in case-control genetic association study, as given in Zheng, Li and Yuan [2], hereafter ZLY, by evaluate their AREs relative to each other. Four commonly used ARE measures, the Pitman ARE, Chernoff ARE, Hodges-Lehmann ARE and the Bahadur ARE are considered. Pitman's ARE does not apply directly. We found the Chernoff, Hodges-Lehmann and the Bahadur AREs are suitable for our setting. Some modifications of these methods are made to simplify the computations.

Existing studies on ARE are mainly focused on two categories. One is to compare efficiencies of estimators of the same parameter; the other is to compare test statistics of the same hypothesis, in which the test statistics may not estimate the same parameter. The latter study can be under the assumption that the test statistics in comparison are asymptotic normality. In this case, the ARE's can often be easily computed. There are also methods for compare ARE of different test statistics in general, in which different test statistics of the same hypothesis may have different asymptotic distributions. In this general case, Pitman, Bahadure and Hodges-Lehmann proposed different ways to compute the ARE, and it is often difficult. Although, when the test statistics have the same asymptotic distribution, the ARE can be computed easily. We also give a simple definition of ARE, so that it can be computed in the case of different asymptotic distributions, as long as the asymptotic distributions of the test statistics are known.

In Section 2, we describe the background of the genetic association study problem and a brief review of the classical definitions of ARE. In Section 3 we compare the ARE of the test statistics arose from our genetic association study. We found that he performances, or the efficiencies of the two test statistic varies for different criterion used, and for different parameter values under the same criterion, which described in the context. Section 4 gives brief numerical examples in simulation and application of the two tests in genetic association study, from our previous study, to illustration their usage.

\section{BACKGROUND}

Denote the log-likelihood function as, $l_{n}\left(\lambda_{1}, \lambda_{2}, \eta\right)=\sum_{i=1}^{n} \log f\left(Y_{i} \mid \lambda_{1}, \lambda_{2}, \eta^{T} X_{i}\right)$ where $Y_{i}$ is the outcome, $\left(\lambda_{1}, \lambda_{2}\right) \in \Lambda \subset R^{2}$ are the parameters of interest, $\eta \in R^{m}$ is a vector of parameters $(\mathrm{m} \geq 0)$ for the covariate $X_{i}=$ $\left(\mathrm{x}_{1, \ldots .,} \mathrm{X}_{\mathrm{im}}\right)^{\mathrm{T}}$, and $\mathrm{n}$ is the sample size. The goal is to test the null hypothesis $H_{0}:\left(\lambda_{1}, \lambda_{2}\right)=(1,1)$ against the alternative $H_{1}\left(\lambda_{1}, \lambda_{2}\right) \Lambda \backslash\{(1,1)\}$, where $\Lambda$ has two edges with known slopes $\theta_{0}$ and $\theta_{1}$, and the null point $(1,1)$ is on the boundary of $\Lambda$. We assume $-\infty<\theta_{0}<\theta_{1}<\infty$ and the endpoints $\theta_{0}$ and $\theta_{1}$ satisfy some constraints as specified in ZLY. If $\theta_{1}=\infty$ which corresponds to a vertical edge, we can switch $\lambda_{1}$ and $\lambda_{2}$ and define new $\left(\theta_{1}, \theta_{2}\right)$ so $-\infty<\theta_{0}<\theta_{1}<\infty$ is satisfied by the new $\left(\theta_{1}, \theta_{2}\right)$. For example, we can write $\lambda_{1}=1+\left(\lambda_{2}-1\right) / \lambda_{1}^{*}\left(\lambda_{2}-1\right)$ and $\lambda_{1}=1+\left(\lambda_{2}-1\right) / \theta_{0}=1+\theta_{0}{ }^{*}\left(\lambda_{2}\right.$ - 1) where $-\infty<\theta_{0}<\theta_{1}<\infty$.

Assume $\theta_{0}$ and $\theta_{1}$ are known from the problem of interest and/or scientific knowledge. Given $\lambda_{1}=\lambda \geq 1, \lambda_{2}$ can be written as $\lambda_{2}=1-\theta+\theta \lambda, \theta \in\left[\theta_{0}, \theta_{1}\right]$. We treat $\eta$ as a nuisance parameter not estimable under $H_{0} \lambda=1$, but

$\square$ it is estimable under. Then the log-likelihood becomes. $l_{n}(\lambda, \eta, \theta)$ The score test statistic $H_{0} \lambda=1$ for is given by;

$$
Z(\theta)=\frac{\left.\frac{\partial}{\partial \lambda} l_{n}(\lambda, \eta, \theta)\right|_{H_{0}, \hat{\eta}_{n}}}{\left\{\operatorname{Var}_{H_{0}, \hat{\eta}_{n}}\left(\frac{\partial}{\partial \lambda} l_{n}(\lambda, \eta, \theta)\right)\right\}^{1 / 2}}
$$

where $\hat{\eta}_{n}$ is the MLE of $\eta$ under $H_{0}$. It would be difficult to deal with $l_{n}(\lambda, \eta, \theta)$ because $\theta$ in $Z(\theta)$ is implicitly expressed.

So we work with $l_{n}(\lambda, 1-\theta+\theta \lambda, \eta)$, where $\theta$ is explicitly expressed. It is convenient to view $l_{n}(\lambda, \eta, \theta)$ as a trivariate function with variables $\mathrm{x}_{1}=\lambda, \mathrm{x}_{2}=1-\theta+\theta \lambda$ and $\mathrm{x}_{3}=\eta$. Denote $l_{n, u}=\partial \mathrm{l}_{\mathrm{n}} / \partial \mathrm{x}_{\mathrm{u}}$ for, $\mathrm{u}=1,2,3,1_{\mathrm{n}, \mathrm{uv}}=\partial^{2} 1_{\mathrm{n}} / \partial \mathrm{x}_{\mathrm{u}} \partial \mathrm{x}_{\mathrm{v}}$ for $\mathrm{u}=1,2$ and, $\mathrm{v}=1.2 .3, \quad$ and $1_{\mathrm{n} .33}=\partial^{2} 1_{\mathrm{n}} / \partial \mathrm{x}_{3} \partial \mathrm{x}_{3}^{\mathrm{T}}$. Assume 
$l_{n, u v}=l_{n, v u}$ for $u, v=1,2, l_{n, u v}=l_{n, v u}^{T}$ for $u=1,2$ and $v=3$. Denote $L_{v u}(\eta)=E_{H n} l_{l v u}(1.1, \eta)$.

Suppose we have a family of asymptotically normally distributed tests $T_{0}=\{Z(\theta): \theta \in[a, b]\}$, where $Z(\theta) \stackrel{D}{\rightarrow} N(0,1)$ under $H_{l} \lambda=1$ for a given $\theta \in[a, b]$, which determines the data-generating model under $\mathrm{H}_{0}: \lambda=1$. When $\theta=\theta^{(0)} \in[a, b]$ is the true value $Z\left(\theta^{0}\right)$, is asymptotically most powerful (optimal). In this case, $\theta^{(1)} \neq \theta^{(0)}$ when is used, the Pitman ARE of $Z\left(\theta^{(1)}\right)$ relative to $Z\left(\theta^{(1)}\right)$ is given by (Gastwirth [3, 4])

$$
e\left(Z\left(\theta^{(1)}\right), Z\left(\theta^{(0)}\right)\right)=\rho_{\theta^{(0)}, \theta^{(1)}}^{2},
$$

where is the asymptotic null correlation coefficient between and. Let be a set of all convex linear combinations of. A simple robust test derived under efficiency robust theory (Gastwirth [3, 4]; Birnbaum and Laska [5],) is the maximin efficient robust test (MERT), denoted as. When, is given by;

$$
Z_{\mathrm{MERT}}=\left(Z\left(\theta_{i}\right)+Z\left(\theta_{j}\right)\right) /\left\{2\left(1+\rho_{\theta_{i}, \theta_{j}}\right)\right\}^{1 / 2} .
$$

When $T_{0}$ has more than two members, generally exists and is unique (Gastwirth [3]), but its computation needs quadratic programming methods (Rosen [6]). However, when there is an extreme pair $\left(Z\left(\theta_{i}\right), Z\left(\theta_{i}\right)\right)$ in $T_{o}$ i.e. $\mathrm{p}_{\theta \mathrm{i}, \theta \mathrm{i}}=$ $\inf _{\theta, \theta^{\prime} \in[a, b]} \rho_{\theta, \theta^{\prime}}>0$, then $Z_{\text {MERT }}=\left(Z\left(\theta_{i}\right)+Z\left(\theta_{j}\right)\right) /\left\{2\left(1+\rho_{\theta_{i}, \theta_{j}}\right)\right\}^{1 / 2}$ is MERT for if and only if (Gastwirth $[7])$.

and thus

$$
\rho_{\theta_{i}, \theta}+\rho_{\theta_{j}, \theta} \geq 1+\rho_{\theta_{i}, \theta_{j}}, \quad \forall \theta \in[a, b]
$$

$$
e\left(Z_{\mathrm{MERT}}, Z\left(\theta^{(0)}\right)\right)=\sup z \in T_{1} \inf \theta \in[a, b] e(Z, Z(\theta)) .
$$

That is, the MERT reaches the maximin ARE due to model uncertainty. The MERT was first derived for linear rank tests for the two-sample problem (Gastwirth [3]; Birnbaum and Laska [5],) and later extended to a family of asymptotically normally distributed tests (Gastwirth [4]).

The $Z(\theta)$ statistic has the following property (ZLY): Let. $\theta \in\left[\theta_{i}, \theta_{j}\right] \subseteq\left[\theta_{0}, \theta_{1}\right]$. Then where and

$$
Z(\theta)=\sum_{l=i, j} W_{l}(\theta) Z\left(\theta_{l}\right),
$$

where $W_{i}(\theta)=\left\{\sigma\left(\theta_{i}\right) / \sigma(\theta)\right\}\left\{\left(\theta_{j}-\theta\right) /\left(\theta_{j}-\theta_{i}\right)\right\}$ and $W_{j}(\theta)=\left\{\sigma\left(\theta_{j}\right) / \sigma(\theta)\right\}\left\{\left(\theta-\theta_{i}\right) /\left(\theta_{j}-\theta_{i}\right)\right\}$.

Let $\hat{\eta}_{0, n}$ be the MLE of $\eta$ under $H_{0}$, and $\left(\hat{\eta}_{1, n}, \hat{\lambda}_{n}\right)$ be that of $(\eta, \lambda)$ under $H_{l}$. For given $\theta$, the $X^{2}$ likelihood ratio test statistic is $T(\theta)=2\left[l_{n}\left(\hat{\lambda}_{n}, 1-\theta+\theta \hat{\lambda}_{n}, \hat{\eta}_{1, n}\right)-l_{n}\left(1,1, \hat{\eta}_{0, n}\right)\right]$. For fixed $\theta$, the number of parameters under $H_{l}$ is just 1 more than that under $H_{0}$, so by Wilk's theorem, under $H_{0}$,

$$
T(\theta) \stackrel{D}{\rightarrow} \chi_{1}^{2}
$$

the chi-squared distribution with one degree of freedom. The likelihood ratio test is also widely used in genetic association studies, its properties, including its ARE is well studied in the literature, so we will not investigate it here.

Let the MLE $\left.\left(\partial l_{n} / \partial \eta\right)\right|_{H_{0}, \hat{\eta}_{n}}=l_{n, 3}\left(1,1, \hat{\eta}_{n}\right)=\mathbf{0}$ here 0 presents a vector of 0 's. Let $\eta_{0}$ be the true value (unknown) of $\eta$ under either $H_{0}$ or $H_{l}$, we define the score function as;

$$
U_{n}\left(1,1, \hat{\eta}_{n}\right)=\left.\frac{\partial l_{n}}{\partial \lambda}\right|_{H_{0}, \hat{\eta}_{n}}=l_{n, 1}\left(1,1, \hat{\eta}_{n}\right)+\theta l_{n, 2}\left(1,1, \hat{\eta}_{n}\right)
$$

and the test statistic for $H_{0}$ as; 


$$
\begin{gathered}
z(\theta)=\frac{U_{n}\left(1,1, \hat{\eta}_{n}\right)}{\left\{\operatorname{Var}_{H_{0}}\left(U_{n}\left(1,1, \hat{\eta}_{n}\right)\right)\right\}^{1 / 2}} \sim \frac{n^{-1 / 2} U_{n}\left(1,1, \hat{\eta}_{n}\right)}{\left\{(1, \mathbf{0}) I^{-1}\left(\eta_{0}\right)(1, \mathbf{0})^{T}\right\}^{-1 / 2}} \\
=\frac{n^{-1 / 2}\left\{l_{n, 1}\left(1,1, \hat{\eta}_{n}\right)+\theta l_{n, 2}\left(1,1, \hat{\eta}_{n}\right)\right\}}{\left(A_{\eta_{0}} \theta^{2}+2 B_{\eta_{0}} \theta+C_{\eta_{0}}\right)^{1 / 2}},
\end{gathered}
$$

where " $\sim$ " means asymptotically equivalent, in the above $I^{-1}\left(\hat{\eta}_{n}\right)$ is replaced by $I^{-1}\left(\eta_{0}\right), A_{\eta}=L_{23}(\eta) L_{33}^{-1}(\eta) L_{32}-L_{22}(\eta), B_{\eta}=L_{13}(\eta) L_{33}^{-1}(\eta) L_{31}(\eta)-L_{12}(\eta)$ and $C_{\eta}=L_{13}(\eta) L_{33}^{-1}(\eta)$ $L_{31}(\eta)-L_{11}(\eta)$. Recall that $L_{v u}(\eta)=E_{H_{0}} l_{1, v u}(1,1, \eta)$, and it is approximated by $\mathrm{n}^{-1} l_{\mathrm{n}, \mathrm{vu}}(1.1, \eta)$.

Denote $W=\left(\begin{array}{cc}1 & 0 \\ W_{0}(\theta) & W_{1}(\theta) \\ 0 & 1\end{array}\right)$. For a vector $v\left(v_{1}, v_{2}, v_{3}\right)^{\mathrm{T}}$, denote $\|v\|=\sum_{i=1}^{3} v_{i}$. Let $f(y \mid \lambda, 1-\theta+\theta \lambda, \eta)$. be the true density of the data $y$. The null model $f(1,1, \eta)$ is and the alternative model is $f(\cdot \mid \lambda, 1-\theta+\theta \lambda, \eta)$. The following notation is also used under $H_{l}$. For fixed, $(\lambda, \theta)$ let;

$$
\eta_{\theta} \stackrel{\Delta}{=} \alpha(\lambda, \theta)=\arg \sup _{\eta} \int f\left(x \mid \lambda, 1-\theta+\theta \lambda, \eta_{0}\right) \log f(x \mid 1,1, \eta) d x
$$

Under $H_{l}$, the empirical version of $\eta_{0}$ is just $\hat{\eta}_{n}$. We denote the Fisher information and its inverse in the blocked forms as;

$$
I\left(\eta_{0}\right)=\left(\begin{array}{cc}
I_{\lambda \lambda} & I_{\lambda \eta} \\
I_{\eta \lambda} & I_{\eta \eta}
\end{array}\right), \quad \operatorname{DgG} I^{-1}\left(\eta_{0}\right)=\left(\begin{array}{cc}
I^{\lambda \lambda} & I^{\lambda \eta} \\
I^{\eta \lambda} & I^{\eta \eta}
\end{array}\right)
$$

Let $\sigma^{2}(\theta)=\left\{(1, \mathbf{0}) I^{-1}\left(\alpha_{0}\right)(1, \mathbf{0})^{T}\right\}^{-1}=\left(I^{\lambda \lambda}\right)^{-1}$,

$$
s(\theta, \eta)=l_{1,1}(1,1, \eta)+\theta l_{1,2}(1,1, \eta)-\left(L_{13}^{T}(\eta)+\theta L_{23}^{T}(\eta)\right) L_{33}^{-1}(\eta) l_{1,3}(1,1, \eta),
$$

$\mu(\lambda, \theta)=E_{H_{1}, \eta_{0}}\left(s\left(\theta, \eta_{\theta}\right)\right), \quad u(\lambda, \theta)={ }^{\Delta} s\left(\theta, \eta_{\theta}\right)-\mu(\lambda, \theta), \quad \tau^{2}(\lambda, \theta)=E_{H_{1}, \eta_{0}}(u(\lambda, \theta))^{2}, \quad \tilde{\sigma}^{2}(\lambda, \theta)=$ $\tau^{2}(\lambda, \theta) / \sigma^{2}(\theta)$, DQG $\widetilde{\Omega}=\left(\widetilde{\omega}_{i j}\right)_{2 \times 2} \quad$ ZIKK $\quad \widetilde{\omega}_{11}=\tilde{\sigma}^{2}\left(\lambda, \theta_{0}\right), \quad \widetilde{\omega}_{22}=\tilde{\sigma}^{2}\left(\lambda, \theta_{1}\right), \quad$ DG $\quad \widetilde{\omega}_{12}=$ $E_{H_{1}, \alpha_{0}}\left\{u\left(\lambda, \theta_{0}\right) u\left(\lambda, \theta_{1}\right)\right\} /\left\{\sigma\left(\theta_{0}\right) \sigma\left(\theta_{1}\right)\right\}$. 7KHHP SIUIFDOMHVIRQRI $\widetilde{\Omega}$ एIVLHHQE $\widetilde{\Omega}_{n}$, ZKHH $\eta_{\theta}$ in $\widetilde{\Omega}$ is

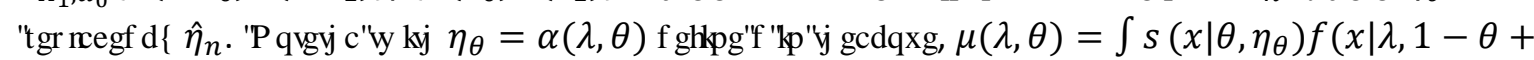
$\left.\theta \lambda, \eta_{0}\right) d x$.

Below we give a brief review of the notions of ARE for test statistics in the general case, more detailed account can be found in Serfling (1980) [8] and Nikitin (2011) [9].

The calculation of the existing of versions of ARE is generally not easy, as in the examples (Serfling, 1980 [8]; Nikitin, 1995 [10]; van der Varrt, 1998 [11]). We only point out that the Pitman ARE is based on the central limit theorem for test statistics, that the Bahadur ARE requires the large deviation asymptotics of test statistics under the nullhypothesis, while the Hodges-Lehmann ARE is connected with large deviation asymptotics under the alternative. Each type of ARE has its own advantage and dis-advantage, and the different notions of ARE are not always give consistent conclusion.

If the condition of asymptotic normality (or common asymptotic distribution) fails, considerable difficulties will arise in calculating the Pitman ARE as it may not at all exist or may depend on $\alpha$ and $\beta$. Usually one considers limiting Pitman ARE as $\alpha \rightarrow 0$ Wieand (1976) [12] established the correspondence between this kind of ARE and the limiting approximate Bahadur efficiency which is easy to compute.

The Bahadur (1960) [13] ARE is to fix the power of tests and compare the exponential rate of decrease of their sizes for the increasing number of observations and fixed alternative. Its computation is always non-trivial, and heavily depends on advancements in large deviation theory, as in Dembo and Zeitouni (1998) [14] and Deuschel and Strook (1989) [15].

It is proved that under some regularity conditions the likelihood ratio statistic is asymptotically optimal in Bahadur sense (Bahadur, 1967 [16]; Arcones, 2005 [17]). Often the Bahadur ARE is difficult to compute for any alternative but it is possible to calculate the limit of Bahadur ARE as $\theta$ approaches the null-hypothesis, to obtain the local Bahadur 
efficiency.

The Hodges-Lehmann ARE is, in contrast to Bahadur efficiency, it fixes the level of tests and compares the exponential rate of decrease of their type-II errors for the increasing number of observations and fixed alternative. The computation of Hodges-Lehmann ARE is also difficult as it requires large deviation asymptotics of test statistics under the alternative.

The drawback of Hodges-Lehmann efficiency is that most two-sided tests like Kolmogorov and Cramer-von Mises tests are all asymptotically optimal, and hence one cannot discriminate among them. On the other hand, under some regularity conditions the one-sided tests, such as linear rank tests can be compared, and their Hodges-Lehmann efficiency coincides locally with Bahadur efficiency (Nikitin, 1995 [10]).

The Chernoff ARE is to minimize, asymptotically, a linear combination of type I and type II errors, it does not depend on the nominal level nor the power. But it basically only applies to test statistics of the form of i.i.d. summation.

The local ARE is much easier to compute than the previous ones, but it only applies to test statistics which are asymptotical normal with rate $\sqrt{n}$. We will see that some test statistics used in genetic association studies do not satisfy this condition.

Besides the four commonly used AREs for hypothesis tests described above, there are some other interesting methods. Hoeffding's (1965) ARE [18], based on the work of Sanov (1957) [19], is theoretically appealing, but ony applies to multinomial data; Rubin and Sethurman ARE (1965) [20] is based on Bayes risk; others including Kallenberg ARE (1983) [21], and the Borovkov-Mogulskii ARE (1993) [22], etc.

\section{ARE OF TWO TESTS IN GENETIC ASSOCIATION STUDIES}

In this section, we investigate the uses of Pitman ARE, Chernoff ARE, Hodges-Lehmman ARE, and Bahadur ARE to the commonly used statistics in genetic association analysis. We focus on the statistics used in ZLY, Z( $\theta)$ and, $Z_{M E R T}$ and refer the notations there. Although some other commonly used test statistics in genetic association studies, such as the likelihood ratio statistic (chi-squared statistic), we will not discuss them here, as most of them are well studied in the literatures.

Pitman ARE. Consider testing $H_{0}: \lambda=\lambda_{0}$ vs $H_{1}: \lambda>\lambda_{0}$. Let $S_{n}$ be a test statistic based on data of size n, with mean $\mu_{\mathrm{n}}(\lambda)$ and standard deviation $\mu_{\mathrm{n}}(\lambda)$. To use this method the following conditions are needed.

(P1). For some continuous strictly increasing distribution function $F$ independent of $\lambda$, and some, $\delta>0$ as $n \rightarrow \infty$,

$$
\sup _{\lambda_{0} \leq \lambda \leq \lambda_{0}+\delta,-\infty<t<\infty} \sup _{-\infty<}\left|P_{\lambda}\left(\frac{S_{n}-\mu_{n}(\lambda)}{\sigma_{n}(\lambda)}\right)-F(t)\right| \rightarrow 0 .
$$

(P2). For $\lambda \in\left[\lambda_{0}, \lambda_{0}+\delta\right], \mu_{n}(\cdot)$, is k times differentiable, with $\mu_{n}^{(1)}\left(\lambda_{0}\right)=\cdots=\mu_{n}^{(k-1)}\left(\lambda_{0}\right)=0<\mu_{n}^{(k)}\left(\lambda_{0}\right)$

$(\mathrm{P} 3)$. For $\mathrm{d}(\mathrm{n}) \rightarrow \infty$ some and some constant $c>0, \sigma_{n}\left(\lambda_{0}\right) \sim c \mu_{n}^{(k)}\left(\lambda_{0}\right) / d(n)$.

(P4). For $\lambda_{n}=\lambda_{0}+O\left(d^{-1 / k}(n)\right), \mu_{n}^{(k)}\left(\lambda_{n}\right) \sim \mu_{n}^{(k)}\left(\lambda_{0}\right)$ and $\sigma_{n}\left(\lambda_{n}\right) \sim \sigma_{n}\left(\lambda_{0}\right)$.

Pitman appears as the first to introduce the notion of ARE for tests in his unpublished lectures, and the following result was stated in Noether's works.

(Pitman, 1949 [23]; Noether, 1950 [24]). Assume (P1)-(P4), that $\alpha_{\mathrm{n}}=\mathrm{P}_{\lambda_{0}}\left(\mathrm{~S}_{\mathrm{n}}>u_{\alpha n}\right) \rightarrow \alpha(0<\alpha<1)$, that $0<\beta<1-\alpha$, and that $\lambda_{n}=\lambda_{0}+O\left(d^{-1 / k}(n)\right)$, then

(i) $\beta_{n}\left(\lambda_{n}\right)=P_{\lambda_{n}}\left(S_{n} \leq u_{\alpha n}\right) \rightarrow \beta$, if and only if

$$
\frac{\left(\lambda_{n}-\lambda_{0}\right)^{k}}{k !} \frac{d(n)}{c} \rightarrow F^{-1}(1-\alpha)-F^{-1}(\beta) .
$$

(ii) Let $S_{l, n}$ and $S_{2, n}$ each satisfy (P1)-(P4) with the common $F, K, n_{1}$ and $n_{2}$ be the sample size required for $S_{l, n}$ and $S_{2, n}$ to have the same asymptotic power $1-\beta$, then 


$$
\frac{d\left(n_{1}\right)}{d\left(n_{2}\right)} \rightarrow \frac{c_{1}}{c_{2}} \text {, with } \quad c_{i}=\lim _{n \rightarrow \infty} \frac{d(n) \sigma_{i, n}\left(\lambda_{0}\right)}{\mu_{i, n}^{(k)}\left(\lambda_{0}\right)} \quad(i=1,2) .
$$

Thus, if $\mathrm{d}(\mathrm{n})=\mathrm{n}^{\mathrm{q}}(\mathrm{q}>0)$, then the Pitman ARE is given by; $e_{P}\left(\left\{S_{1, n}\right\},\left\{S_{2, n}\right\}\right)=\left(c_{2} / c_{1}\right)^{1 / q} .7 \backslash$ SIFDD, $k=1, q$

$=1 / 2, \sigma_{n}(\lambda)=\sqrt{n} \sigma(\lambda), \mu_{n}^{(1)}=n \mu(\lambda)$, then

$$
c=\frac{\sigma\left(\lambda_{0}\right)}{\mu^{(1)}\left(\lambda_{0}\right)}
$$

and Pitman ARE is then;

$$
e_{P}\left(\left\{S_{1, n}\right\},\left\{S_{2, n}\right\}\right)=\left(\frac{c_{2}}{c_{1}}\right)^{2} .
$$

Let $l\left(\lambda_{0}\right)$ be the Fisher information at $\lambda_{0}$. Under some additional conditions, Rao (1963) [25] proved that

$$
\left(\frac{\sigma\left(\lambda_{0}\right)}{\mu^{(1)}\left(\lambda_{0}\right)}\right)^{2} \geq I^{-1}\left(\lambda_{0}\right)
$$

Any test statistic $S_{n}$ achieves the equality in the above is called Pitman efficient.

Under suitable conditions, Pitman ARE can be expressed in terms of correlation coefficient between the two test statistics in their standardized form, as given below.

(P5) $\left(S_{1, n}-\mu_{1, n}(\lambda)\right) / \sigma_{1, n}(\lambda)$ and $\left(S_{2, n}-\mu_{2, n}(\lambda)\right) / \sigma_{2, n}(\lambda)$ are asymptotic joint normal uniformly in a neighborhood of $\lambda$.

Denote $p(\lambda)$ the asymptotic correlation coefficient between them under, and and be the distribution and density function of. The following result is true.

(van Eden, 1963 [26]). Assume that $S_{1, n}$ and $S_{2, n}$ satisfy (P1)-(P5) in their standardized form with $H=\Phi, k=1$ and $d(n)=n^{1 / 2}$, and that $\mathrm{p}\left(\lambda_{\mathrm{n}}\right) \rightarrow \mathrm{p}\left(\lambda \lambda_{\mathrm{n}}\right):=\mathrm{p}$ as $\lambda_{\mathrm{n}} \rightarrow \lambda_{0}$ Then;

(i) For $0 \leq \lambda \leq 1$, tests of the form $S_{\gamma, n}=(1-\gamma) S_{1, n}+\gamma S_{2, n}$ satisfy (P1)-(P5), and the "best" $\mathrm{S}_{\mathrm{yn}}$ which maximizes $e_{P}\left(\left\{S_{\lambda, n}\right\},\left\{S_{1, n}\right\}\right)$ is the one with;

$$
\gamma=\frac{c_{1}-\rho c_{2}}{(1-\rho)\left(c_{1}+c_{2}\right)}=\frac{e_{P}^{1 / 2}\left(\left\{S_{2, n}\right\},\left\{S_{1, n}\right\}\right)-\rho}{(1-\rho)\left[1+e_{P}^{1 / 2}\left(\left\{S_{2, n}\right\},\left\{S_{1, n}\right\}\right)\right]}
$$

and

$$
e_{P}\left(\left\{S_{\gamma, n}\right\},\left\{S_{1, n}\right\}\right)=1+\frac{\left[e_{P}^{1 / 2}\left(\left\{S_{2, n}\right\},\left\{S_{1, n}\right\}\right)\right]^{2}}{1-\rho^{2}} .
$$

(ii) If $S_{I n}$ is the best test satisfying (P1)-(P5), then;

$$
e_{P}\left(\left\{S_{2, n}\right\},\left\{S_{1, n}\right\}\right)=\rho^{2} .
$$

In the typical case, $S_{n}$ is an i.i.d. summation (upto scale), then $\mu_{\mathrm{n}}(\lambda)=n \mu(\lambda)$ $\sigma_{n}(\lambda)=\sqrt{n} \sigma(\lambda), d(n)=\sqrt{n}, k=1, c=\sigma\left(\lambda_{0}\right) / \mu^{\prime}\left(\lambda_{0}\right)$ and $\lambda_{n}=\lambda_{0}+n^{-1 / 2} \sigma\left(\lambda_{0}\right) / \mu^{\prime}\left(\lambda_{0}\right)\left[F^{-1}(1-\alpha)\right.$ $\left.-F^{-1}(\beta)\right]$.

Note $e_{P}\left(\left\{S_{1, n}\right\},\left\{S_{2, n}\right\}\right)$ does not $(\alpha, \beta)$ depend on, thus if $e_{P}\left(\left\{S_{1, n}\right\},\left\{S_{2, n}\right\}\right)<1$ or, $\mathrm{C}_{1}>\mathrm{C}_{2}$ then $\left\{\mathrm{S}_{1 \mathrm{n}}\right\}$ is better than $\left\{\mathrm{S}_{2 \mathrm{n}}\right\}$ for all $(\alpha, \beta)$.

Pitman ARE given by (3) or (4) are easy to use. However, they require the two comparing test statistics have the same asymptotic distribution (after standardization), (4) require further that they are jointly asymptotic normal. In 
practice, these conditions some times cannot be satisfied. For example the chi-squared test $Z\left(\theta_{0}\right)$ and have different asymptotic distributions. Below we give a generalized version of (3) to the case the two comparing test statistics not necessarily have the same asymptotic distribution (after standardization). Similar generalizations may have already exist in the literature, we still state our version to see what form it has in this case. Let $F_{i}$ be the asymptotic distribution of $\left(S_{i, n}-\mu_{i, n}(\lambda)\right) / \sigma_{i, n}(\lambda)$ We have;

Assume (P1)-(P4) for $S_{i n}$ with $\mu_{i n}, \sigma_{\text {in }}$ and $F_{i}$ separately, but with the same $K$ and nominal level $\alpha, n_{1}$ and $n_{2}$ be the sample sizes required for $S_{1 n}$ and $N_{2 n}$ to have the same asymptotic power $1-\beta(0<\beta<1-\alpha)$, then

$$
\frac{d\left(n_{1}\right)}{d\left(n_{2}\right)} \rightarrow \frac{\tilde{c}_{1}}{\tilde{c}_{2}}, \quad \text { with } \quad \tilde{c}_{i}=\lim _{n \rightarrow \infty} \frac{d(n) \sigma_{i, n}\left(\lambda_{0}\right)\left[F_{i}^{-1}(1-\alpha)-F_{i}^{-1}(\beta)\right]}{\mu_{i, n}^{(k)}\left(\lambda_{0}\right)} \quad(i=1,2) .
$$

Thus for $d(\mathrm{n})=\mathrm{n}^{\mathrm{q}}(\mathrm{q}>0)$, we define the generalized Pitman ARE as;

$$
\tilde{e}_{P}\left(\left\{S_{1, n}\right\},\left\{S_{2, n}\right\}\right)=\left(\frac{\tilde{c}_{2}}{\tilde{c}_{1}}\right)^{1 / q} .
$$

In the typical case $\mu_{i, n}=n \mu_{i}, \sigma_{i, n}=\sqrt{n} \sigma_{i}, k=1$ and $d(n)=\sqrt{n}$ or $1 / \mathrm{q}=2$, and;

$$
\tilde{e}_{P}\left(\left\{S_{1, n}\right\},\left\{S_{2, n}\right\}\right)=\left(\frac{\tilde{c}_{2}}{\tilde{c}_{1}}\right)^{2}, \quad \tilde{c}_{i}=\frac{\sigma_{i}\left(\lambda_{0}\right)\left[F_{i}^{-1}(1-\alpha)-F_{i}^{-1}(\beta)\right]}{\mu_{i}^{(1)}\left(\lambda_{0}\right)} \quad(i=1,2) .
$$

Note, unlike the case of $F_{1}=F_{2}$, in this case, Pitman's ARE depends on the values of level $\alpha$ and power $\beta$, and comparison of two tests may not have consistent result.

Can we have the corresponding form of (10) in the case $S_{I n}$ and $S_{2 n}$ have different asymptotic distribution? For this we checked the proof for (4), and find in this case, although in principle there is a relationship among the asymptotic correlation coefficient $\mathrm{p}$ between $S_{I n}$ and $S_{2 n}$, the asymptotic distributions's, $F_{i}{ }^{\prime} s$, and the level $\alpha$ and power $\beta$, but its mathematically intractable. Below we give its actual value.

\section{Proposition 1.}

$$
e_{P}\left(Z_{M E R T}, Z\left(\theta^{(0)}\right)\right)=\frac{\left(\rho_{\theta_{i}, \theta^{(0)}}+\rho_{\theta_{j}, \theta^{(0)}}\right)^{2}}{2\left(1+\rho_{\theta_{i}, \theta_{j}}\right)} .
$$

Remark: When some of the conditions (P1)-(P5) are not satisfied, ARE may not be characterized by correlation coefficient. For example, $T_{l}=Z$ is an estimate of $\theta=0$ under $H_{0}$, and $\mathrm{Z}$ is symmetrically distributed around 0 , so $E_{H o}$ $(Z)=0$ and suppose $V A R_{H o}(Z)=1$. Let, $T_{2}=|Z|, T_{2}$ is an estimate of $E_{H_{0}}(|Z|) \neq 0 . T_{2}$ can also be used to test $H_{0}$. However $\operatorname{Cov}_{H_{0}}(|Z|, Z)=E_{H_{0}}(|Z| Z)-E_{H_{0}}(|Z|) E_{H_{0}}(Z)=0$, but we cannot say that $T_{2}$ is a 'bad' test statistic, and $e_{P}\left(T_{1}, T_{2}\right) \neq \operatorname{Cov}_{H_{0}}^{2} /\left[\operatorname{Var}_{H_{0}}\left(T_{1}\right) \operatorname{Var}_{H_{0}}\left(T_{2}\right)\right]$.

Chernoff ARE. This notion only considers test statistic of the form $S_{n}=\sum_{i=1}^{n} Y_{i}$ with the $\mathrm{s}$ i.i.d. with $Y \sim F$. Let $M(z)=E_{F}\left(e^{z Y}\right)$ be the moment generating function of $Y$, and;

$$
m(t)=\inf _{z} E\left(e^{z(Y-t)}\right)=\inf _{z} e^{-z t} M(z) .
$$

Let $\mu_{0}=E\left(Y \mid H_{0}\right)$ and $\mu_{1}=E\left(Y \mid H_{1}\right)$ (assume $\left.\mu_{0} \leq \mu_{1}\right), m_{i}(t)=\inf _{z} E\left(e^{z(Y-t)} \mid H_{i}\right)=\inf \left[e^{-z t} M_{i}(z)\right], \quad(i=$ $0,1), \rho(t)=\max \left\{m_{0}(t), m_{1}(t)\right\}$ and $\rho=\inf _{\mu_{0} \leq t \leq \mu_{1}} \rho(t)$ called the Chernoff index of $\left\{S_{n}\right\}$. For $0 \leq \gamma<\infty$, let $Q_{n}$ $(t)=P\left(S_{n} \leq n t \mid H_{1}\right)+\gamma P\left(S_{n}>n t \mid H_{0}\right)$ be a linear combination of type I and type II errors evaluated at the critical value $\mathrm{t}$, and $\mathrm{Q}_{\mathrm{n}}=\inf _{\mu 0 \leq \mathrm{t} \leq \mu} \mathrm{Q}_{\mathrm{n}}(\mathrm{t})$ be the minimum of these errors for test statistic $S_{n}$. Chernoff (1952) [27] showed that $Q_{n}$ tends to 0 at exponential rate, (so the faster the rate, or the larger absolute value of $\log \mathrm{Q}_{\mathrm{n}}$, the better the test statistic), and established. 


$$
\lim _{n} n^{-1} \log Q_{n}=\log \rho
$$

the result is independent of $\gamma$.

Let $\left\{\mathrm{S}_{1, \mathrm{n}}\right\}$ and $\left\{\mathrm{S}_{2, \mathrm{n}}\right\}$ both of the form of i.i.d. summation and have Chernoff indices $p_{1}$ and $p_{2}$ respectively, $n_{l}$ and $n_{2}$ be the corresponding sample sizes for which $\mathrm{Q}_{1, \mathrm{n}}, \sim \mathrm{Q}_{2, \mathrm{n}}$, the Chernoff ARE of $\left\{\mathrm{S}_{1, \mathrm{n}}\right\}$ relative to $\left\{\mathrm{S}_{2, \mathrm{n}}\right\}$ is defined and given by;

$$
e_{C}\left(\left\{S_{1, n}\right\},\left\{S_{2, n}\right\}\right)=\lim \frac{n_{2}}{n_{1}}=\frac{\log \rho_{1}}{\log \rho_{2}} .
$$

For test statistic not in the form of i.i.d summation, its Chernoff index is difficult to compute. The following result sometimes is very helpful in this case, and give an upper bound of Chernoff index.

(Kallenberg, 1982 [28]) Let for some $t \in R^{1}$, and $\lambda \in \Lambda_{1}$

$$
\lim _{n \rightarrow \infty} \log \sup \left\{P_{\lambda_{0}}\left(S_{n}>n t\right): \lambda_{0} \in \Lambda_{0}\right\}=\lim _{n \rightarrow \infty} \inf \log P_{\lambda}\left(S_{n} \leq n t\right):=u(\lambda)
$$

Then $\rho(\lambda)=-u(\lambda)$.

In the case of simple null $v s$ simple alternative, Kallenberg (1982) [28] also gives an upper bound of the Chernoff index, and any test statistic achieves this bound is said to be Chernoff efficient. As this bound itself is not easy to compute, we won't pursue it here, interested readers can check the mentioned paper or the book by Nikitin (1995) [10].

As another way to simplify the computation, we consider a modified version of this Chernoff index. Let $\mathrm{S}$ be the weak limit of $S_{n}$, be the distribution function of $S$, and $H_{n}: \lambda_{n}+\lambda_{n}=n^{-1 / 2}$ be a sequence of local alternatives. As the sample size increases, the test statistic $S_{n}$ is expected to be able to distinguish the local alternatives from the null. Let $\mu_{0}=E\left(S \mid H_{0}\right), \mu_{1}=\lim _{n} E\left(S \mid H_{n}\right)$ (assume $\left.\mu_{1} \geq \mu_{0}\right)$, and $\tilde{Q}(t)=\lim _{n} G\left(S \leq t \mid H_{n}\right)+G\left(S>t \mid H_{0}\right)$ be the asymptotic linear combination of type I and local type II errors evaluated at t, and $\tilde{Q}=\inf _{\mu_{0} \leq t \leq \mu_{1}} \tilde{Q}(t)$. The smaller is $\tilde{Q}$, the better $S_{n}$ as a test statistic for $H_{0} v s . H_{l}$ For two test statistics $S_{l n}$ and $S_{2 n}$ with $\widetilde{Q}_{1}$ and $\tilde{Q}_{2}$, we define the modified Chernoff ARE as;

$$
\tilde{e}_{C}\left(\left\{S_{1, n}\right\},\left\{S_{2, n}\right\}\right)=\frac{\tilde{Q}_{2}}{\tilde{Q}_{1}}
$$

Let $\mu^{(1)}(\lambda, \theta)=\partial \mu(\lambda, \theta) / \partial \lambda$, and

$$
\zeta^{(1)}=\left(\zeta_{1}^{(1)}, \ldots, \zeta_{k}^{(1)}\right)^{T}=\left(\frac{\mu^{(1)}\left(\lambda_{0}, a_{1}\right)}{\sigma\left(a_{1}\right)}, \ldots, \frac{\mu^{(1)}\left(\lambda_{0}, a_{k}\right)}{\sigma\left(a_{k}\right)}\right)^{T} .
$$

Below we give values $p_{z(\theta(0))}$ and $\mathrm{p}_{z_{\text {Narr }}}$ and so that their Chernoff ARE can be obtained. We also give and, so their modified Chernoff ARE can be obtained. For the chi-squared test $T$, under $T_{l}$ its asymptotic distribution is a non-central chi-squared distribution, with a non-closed form, its modified Chernoff index is not directly computable. Let $b\left(g_{i}\right)=\log (1-\theta+\theta \lambda) I_{1}\left(g_{i}\right)+\log (\lambda) I_{2}\left(g_{i}\right)$, where $g_{l}$ is the observed genotype of the $i$-th individual, $x_{1}$ is the corresponding covariates, and let;

$$
a\left(\boldsymbol{x}_{i}, g_{i}, \lambda\right)=\frac{I_{1}\left(g_{i}\right)}{(1-\theta+\theta \lambda) \sigma^{2}}+\frac{I_{2}\left(g_{i}\right)}{\lambda \sigma^{2}}+\frac{\left(L_{13}^{T}\left(\eta_{0}\right)+\theta L_{23}^{T}\left(\eta_{0}\right)\right) L_{33}^{-1}\left(\eta_{0}\right) \boldsymbol{x}_{i}}{\sigma^{2}} .
$$

$$
\begin{aligned}
& \text { Let } b_{1}\left(g_{r}\right)=\left[\log (1-\theta+\theta \lambda) I_{1}\left(g_{r}\right)+\log (\lambda) I_{2}\left(g_{r}\right)\right] / \sqrt{2\left(1+\rho_{\theta_{i}, \theta_{j}}\right)} \text {, and } a_{1}\left(\boldsymbol{x}_{r}, g_{r}\right)= \\
& {\left[a\left(\boldsymbol{x}_{r}, g_{r}, \theta_{i}, \lambda\right)+a\left(\boldsymbol{x}_{r}, g_{r}, \theta_{j}, \lambda\right)\right] / \sqrt{2\left(1+\rho_{\theta_{i}, \theta_{j}}\right)} .}
\end{aligned}
$$

Proposition 2. (i) Assume $Y \mid(x, g)$ is normal with mean $\eta^{T} \boldsymbol{x}+\sum_{j=1}^{2} \log \left(\lambda_{j}\right) I_{j}(g)$ and variance $\sigma^{2}, \lambda_{1}=\lambda$, and $\lambda_{2}=1-\theta+\theta \lambda$. Then, for $E$ to denote expectation with respect to $\left(x_{i}, g_{i}\right)$, we have; 


$$
\begin{aligned}
\rho_{Z(\theta)} & =E\left(\exp \left[-\frac{b^{2}\left(g_{i}\right)}{2\left(a\left(\boldsymbol{x}_{i}, g_{i}, 1\right)+a\left(\boldsymbol{x}_{i}, g_{i}, \lambda\right)\right)^{2}}\right]\right), \\
\rho_{Z_{M E R T}} & =E\left(\exp \left[-\frac{b_{1}^{2}\left(g_{i}\right)}{2\left(a_{1}\left(\boldsymbol{x}_{i}, g_{i}, 1\right)+a_{1}\left(\boldsymbol{x}_{i}, g_{i}, \lambda\right)\right)^{2}}\right]\right) .
\end{aligned}
$$

(ii)

$$
\begin{gathered}
\tilde{Q}_{Z\left(\theta^{(0)}\right)}=2\left(1-\Phi\left(\frac{\mu^{(1)}\left(\lambda_{0}, \theta^{(0)}\right)}{2 \sigma\left(\theta^{(0)}\right)}\right)\right), \\
\tilde{Q}_{Z_{M E R T}}=2\left(1-\Phi\left(\left[\frac{\mu^{(1)}\left(\lambda_{0}, \theta_{i}\right)}{\sigma\left(\theta_{i}\right)}+\frac{\mu^{(1)}\left(\lambda_{0}, \theta_{j}\right)}{\sigma\left(\theta_{j}\right)}\right] / \sqrt{8\left(1+\rho_{\theta_{i}, \theta_{j}}\right)}\right)\right) .
\end{gathered}
$$

Hodges-Lehmann ARE. Consider testing the null hypothesis be $H_{0}: \lambda \in \Lambda_{0}$ vs $H_{1}: \lambda \in \Lambda_{1}$, given a level $\alpha$ test statistic $S_{n}$ with critical value $t_{n}(\alpha): \alpha_{n}:=\sup _{\lambda \in \Lambda_{0}} P_{\lambda}\left(S_{n} \geq t_{n}(\alpha)\right) \rightarrow \alpha$. For $\lambda \in \Lambda_{1}$, the type II error at $\lambda$ is $\beta_{n}(\lambda)=P_{\lambda}\left(S_{n} \leq t_{n}(\alpha)\right)$. Typically, $\beta_{n}(\lambda)$ tends to zero at exponential rate, the faster the better $S_{n}$ is. Hodges and Lehmann (1956) [29] proposed;

$$
d(\lambda)=\lim _{n}-2 n^{-1} \log \beta_{n}(\lambda)
$$

as a measure of the performance of $S_{n}$ and it called the Hodges-Lehmann index of the statistic $S_{n}$. For two test statistics $S_{I n}$ and $S_{2 n}$ for the same $H_{0} v s, H_{1}$ with $d_{l}(\lambda)$ and $d_{2}(\lambda)$, the Hodges-Lehmann ARE of $\left\{S_{I n}\right\}$ relative to $\left\{S_{2 n}\right\}$ at $\lambda \in \Lambda_{1}$ is defined as;

$$
e_{H L}\left(\left\{S_{1, n}\right\},\left\{S_{2, n}\right\}\right)=\frac{d_{1}(\lambda)}{d_{2}(\lambda)}
$$

For probability density functions $f$ and $g$, let $K(f, g)=\int f(x) \log [f(x) / g(x)] d x$ be the Kullback-Leibler divergence between $f)$ and $g$ ). For any test statistic $S_{n}\left(X_{1}, \ldots \ldots, X_{n}\right)$ based on $\left(X_{1}, \ldots \ldots, X_{n}\right)$ i.i.d. density $f(\cdot \mid \lambda)$, the HodgesLehmann index has the following property;

$$
\lim _{n}\left(1-\beta_{n}(\lambda)\right) \geq-\inf \left\{K\left(f\left(\cdot \mid \lambda_{0}\right), f(\cdot \mid \lambda)\right): \lambda_{0} \in \Lambda_{0}\right\}
$$

and any test statistic achieve the equality in the above is said to be Hodges-Lehmann efficient.

Compared to the Pitman and Chernoff ARE, the Hodges-Lehmman ARE does not require the comparing test statistic have the same asymptotic distribution, nor they have the form of i.i.d. summations, so it has wilder application scope.

Proposition 3. Under conditions of Theorem 4 in Zheng et al. (2010) [30], with $\mu_{M E R T}(\lambda):=\left[\mu\left(\lambda, \theta_{i}\right) / \sigma\left(\theta_{i}\right)+\mu\left(\lambda, \theta_{j}\right) / \sigma\left(\theta_{j}\right)\right] / \sqrt{2\left(1+\rho_{\theta_{i}, \theta_{j}}\right)}$, and $\tilde{\zeta}$, given in (2), for $\lambda>1$, we have;

$$
d_{Z(\theta)}(\lambda)=\frac{\mu^{2}(\lambda, \theta)}{\sigma^{2}(\theta)}, \quad d_{Z_{M E R T}}(\lambda)=\mu_{M E R T}^{2}(\lambda) .
$$

For the chi-squared test $T$, under $H_{l}$ its asymptotic distribution is a non-central chi-squared distribution, with noclosed form. So its Hodges-Lehmann ARE is not directly available.

Bahadur ARE. Consider testing the null hypothesis be $H_{0}: \lambda \in \Lambda_{0}$ vs $H_{1}: \lambda \in \Lambda_{1}$. Let $F_{n, \lambda}($.$) be the distribution$ function of a test statistic $S_{n}$ under $p_{\lambda}$, and for $\lambda \in \Lambda_{1}$, let;

$$
L_{n}(\lambda)=\sup _{\xi \in \Lambda_{0}}\left[1-F_{n, \xi}\left(S_{n} \mid \lambda\right)\right],
$$

the p-value of the observed $S_{n}$ under the distribution $p_{\lambda}$, and; 


$$
c(\lambda)=\lim _{n \rightarrow \infty}\left(-2 n^{-1} \log L_{n}(\lambda)\right)
$$

if the limit exists. Typically, $L_{n}$ tends to one and $L_{n}$ tends to zero exponentially fast, and the faster, or the bigger c(.), the better $S_{n}$ is. For two test statistics $S_{i, n}(1=1,2)$ for the same hypothesis with $L_{n}, \mathrm{C}_{\mathrm{i}}(\lambda)$, and sample size $n_{i}$, to perform "equivalently" in the sense $\lim n_{1}^{-1} \log L_{2, n_{2}}=\lim n_{1}^{-1} \log L_{1, n_{1}}$, the Bahadur ARE of $S_{l, n} \log L_{l n}$, the Bahadur ARE of relative to $S_{2, n}$, at $\lambda \in \Lambda_{1}$, is defined as, and has the property

$$
e_{B}\left(\left\{S_{1, n}\right\},\left\{S_{2, n}\right\}\right)=\lim \frac{n_{2}}{n_{1}}=\frac{c_{1}(\lambda)}{c_{2}(\lambda)} .
$$

The limit $C$ can be computed under the following conditions.

(B1). For $\lambda \in \Lambda_{1}, n^{-1 / 2} S_{n} \rightarrow b(\lambda)$ a.s. $\left(P_{\lambda}\right)$, for some $-\infty<b(\lambda)<\infty$.

(B2). For the interval $I=\left\{b(\lambda): \lambda \in \Lambda_{1}\right\}$, there is a function $g$ on $l$, such that;

$$
\lim _{n}-2 n^{-1} \log \sup _{\xi \in \Lambda_{0}}\left[1-F_{n, \xi}\left(n^{1 / 2} t\right)\right]=g(t), \quad t \in I .
$$

(Bahadur, 1960 [13]). If $S_{n}$ satisfies (B1)-(B2), then for $\lambda \in \Lambda_{1}$,

$$
c(\lambda)=g(b(\lambda)) \text { a.s. }\left(P_{\lambda}\right) .
$$

For any test statistic $S_{n}\left(X_{1}, \ldots, X_{2}\right)$ based on $X_{1}, \ldots, X_{n}$ i.i.d. density $f(\cdot \mid \lambda)$, Bahadur (1967) [16] obtained the following;

$$
\lim _{n} n^{-1} \log L_{n}(\lambda) \geq-\inf \left\{K\left(f(\cdot \mid \lambda), f\left(\cdot \mid \lambda_{0}\right)\right): \lambda_{0} \in \Lambda_{0}\right\} .
$$

Note although the above relationship is regarded as a dual to that of the Hodges-Lehmann index, the two are not equivalent as $K\left(f(\cdot \mid \lambda), f\left(\cdot \mid \lambda_{0}\right)\right) \neq K\left(f\left(\cdot \mid \lambda_{0}\right), f(\cdot \mid \lambda)\right)$. A test statistic is said to be Bahadur efficient if for each $\lambda \in \Lambda_{1} \quad \lim _{n}, \log L_{n}(\lambda)=-\inf \left\{K\left(f(\cdot \mid \lambda), f\left(\cdot \mid \lambda_{0}\right)\right): \lambda_{0} \in \Lambda_{0}\right\}$.

Bahadur efficiency of likelihood ratio test has been studied by a number of researchers for some special distribution families. Arcones (2005 [17], Theorem 3.3) proved that, under some regularity conditions, the likelihood ratio statistic is Bahadur efficient. Let $f(\cdot \mid \lambda, \theta, \eta)$ be the density function of the data, under his conditions of Theorem 3.3, for each fixed $\lambda>1$ and $\theta$, we have;

$$
c_{T}=-2 \inf \inf \left\{K\left(f(\cdot \mid \lambda, \theta, \eta), f\left(\cdot \mid \eta, \lambda_{0}\right)\right): \eta\right\} .
$$

Like the Hodges-Lehmman ARE, Bahadur ARE does not require the comparing test statistic have the same asymptotic distribution, nor they have the form of i.i.d. summations, so it has wide application scope.

For computation easiness, we consider a local version of Bahadur ARE. Consider testing $H_{0}: \lambda=\lambda_{0} v s$ the local alternative $H_{0}: \lambda=\lambda_{0}+n^{-1 / 2}$. Let $F_{0}$ be the asymptotic distribution function of $S_{n}$ under $H_{0}$, we define;

$$
\tilde{c}=\lim _{n}\left[1-F_{0}\left(S_{n} \mid H_{n}\right)\right] .
$$

Typically, $0<\tilde{c}<1$. The smaller $\tilde{c}$, the better $S_{n}$ is. For two test statistics $S_{i, n}(i=1,2)$ for the same hypothesis with $G_{i, n}$ and $\tilde{c}_{i}$, we define the local Bahadur ARE of $S_{l, n}$ relative to $S_{2, n}$ as;

$$
\tilde{e}_{B}\left(\left\{S_{1, n}\right\},\left\{S_{2, n}\right\}\right)=\frac{\tilde{c}_{2}}{\tilde{c}_{1}} .
$$

Proposition 4. (i) with $\mu_{\text {MERT }}(\lambda)$ given in Proposition 3, we have;

$$
c_{Z(\theta)}(\lambda)=\mu^{2}(\lambda, \theta) / \sigma^{2}(\theta), \quad c_{Z_{M E R T}}(\lambda)=\mu_{M E R T}^{2}(\lambda) .
$$

(ii) Under conditions of Theorem 4 in $Z L Y, \mu_{M E R T}(\lambda)$ with be the derivative of $\mu_{M E R T}(\lambda), \theta_{0}$ be the value of $\theta H_{0}$ 
under, we have;

$$
\tilde{c}_{Z(\theta)}=1-\Phi\left(\mu^{(1)}\left(1, \theta_{0}\right) / \sigma\left(\theta_{0}\right)\right), \quad \tilde{c}_{Z_{M E R T}}=1-\Phi\left(\mu_{M E R T}^{(1)}(1)\right) .
$$

\section{SIMULATION AND APPLICATION TO GENETIC ASSOCIATION STUDIES}

\subsection{Simulation Study}

Let $P$ be the Minor Allele Frequency (MAF) of a marker of interest. We consider case-control data with $\mathrm{r}=500$ cases and $s=500$ controls, $\lambda \in\{1.1,1.3,1.5\}, p \in\{0.15,0.30,0.45\}$, the true $\theta^{(0)} \in\{1 / 2,1\}$, and the disease prevalence $K$ $=0.05$. We generate 1000 datasets, and compute the means and standard deviations of $e_{P}\left(Z_{\mathrm{MERT}}, Z_{\theta^{(0)}}\right), \tilde{e}_{C}\left(Z_{\mathrm{MERT}}, Z_{\theta^{(0)}}\right)$ and $\tilde{e}_{B}\left(Z_{\mathrm{MERT}}, Z_{\theta^{(0)}}\right)$. For $Z_{M E R T}$, we choose $\theta_{\mathrm{i}}=0$ and $\theta_{\mathrm{j}}=1$.

Table T1 shows the result, the means of AREs and the standard deviations of AREs are in brackets. First we can see the mean of all three AREs are less than 1, which show that $Z_{\theta}$ is consistent better than $Z_{M E R T}$. Corresponding tothis fact when $\theta=\theta_{(\circ)}$ is the true value $Z_{\theta^{(o)}}$, is asymptotically most powerful. Then the three AREs are increased with the $P$ or $\lambda$ increased. Third, the $e_{p}$ has the lowest variance among the three AREs, next is $\widehat{l}_{\text {lde }} e_{C}$, (DWWV $\tilde{e}_{B}$.

Table 1. The AREs of $Z_{\mathrm{MERT}}$ and $Z_{\theta(0)}$.

\begin{tabular}{|c|c|c|c|c|c|c|c|c|c|c|}
\hline- & - & \multicolumn{3}{|c|}{$\lambda-\mathbf{1 . 1}$} & \multicolumn{3}{|c|}{$\lambda-\mathbf{1 . 1}$} & \multicolumn{3}{c|}{$\lambda-\mathbf{1 . 1}$} \\
\hline $\mathbf{M A F}_{\mathbf{0}}$ & $\boldsymbol{\theta}(\mathbf{0})$ & $\boldsymbol{e p}$ & $\tilde{e}_{C}$ & $\tilde{e}_{B}$ & $\boldsymbol{e p}$ & $\tilde{e}_{C}$ & $\tilde{e}_{B}$ & $\boldsymbol{e p}$ & $\tilde{e}_{C}$ & $\tilde{e}_{B}$ \\
\hline 0.15 & $1 / 2$ & 0.874 & 0.876 & 0.827 & 0.887 & 0.904 & 0.856 & 0.895 & 0.917 & 0.869 \\
\hline- & - & $(0.056)$ & $(0.1)$ & $(0.115)$ & $(0.048)$ & $(0.084)$ & $(0.11)$ & $(0.039)$ & $(0.069)$ & $(0.097)$ \\
\hline- & 1 & 0.654 & 0.814 & 0.723 & 0.654 & 0.837 & 0.746 & 0.652 & 0.85 & 0.761 \\
\hline- & - & $(0.037)$ & $(0.094)$ & $(0.101)$ & $(0.031)$ & $(0.084)$ & $(0.094)$ & $(0.029)$ & $(0.075)$ & $(0.092)$ \\
\hline 0.3 & $1 / 2$ & 0.963 & 0.94 & 0.912 & 0.97 & 0.954 & 0.929 & 0.973 & 0.961 & 0.937 \\
\hline- & - & $(0.018)$ & $(0.05)$ & $(0.069)$ & $(0.013)$ & $(0.042)$ & $(0.064)$ & $(0.011)$ & $(0.039)$ & $(0.061)$ \\
\hline- & 1 & 0.73 & 0.841 & 0.751 & 0.729 & 0.853 & 0.763 & 0.728 & 0.863 & 0.775 \\
\hline- & - & $(0.03)$ & $(0.045)$ & $(0.056)$ & $(0.028)$ & $(0.043)$ & $(0.055)$ & $(0.025)$ & $(0.037)$ & $(0.05)$ \\
\hline 0.45 & $1 / 2$ & 0.991 & 0.985 & 0.978 & 0.993 & 0.986 & 0.978 & 0.995 & 0.989 & 0.983 \\
\hline- & - & $(0.006)$ & $(0.038)$ & $(0.055)$ & $(0.004)$ & $(0.036)$ & $(0.054)$ & $(0.003)$ & $(0.032)$ & $(0.051)$ \\
\hline- & 1 & 0.76 & 0.85 & 0.766 & 0.758 & 0.856 & 0.771 & 0.76 & 0.861 & 0.775 \\
\hline- & - & $(0.032)$ & $(0.033)$ & $(0.044)$ & $(0.031)$ & $(0.031)$ & $(0.042)$ & $(0.027)$ & $(0.028)$ & $(0.039)$ \\
\hline
\end{tabular}

\subsection{Application}

We use 6 reported SNPs associated with breast cancer 2 (Hunter et al. 2007 [31]; Li et al., 2008 [32]) to illustrate the ARE of $Z_{M E R T}$. These 6 SNPs are rs10510126, rs12505080, rs17157903, rs1219648, rs7696175, and rs2420946. The counts of subjects with three types of genotypes in cases and controls are shown in Table 2, where $\left(r, r_{1}, r_{2}\right)$ is the number of three genotypes in cases and $\left(s, s_{1}, s_{2}\right)$ is the number of genotypes in controls. From the table, we find three AREs of $E_{p}, E_{c}$ and $E_{b}$ are higher than $75 \%$, sometimes it can reach 97\%. For example, for SNP rs 17157903 , the AREs of, and are $0.8255,0.8453$ and 0.7642 , respectively. It shows that $Z_{M E R T}$ is a robust test.

Table 2. Three AREs of for 6 reported SNPs associated with breast cancer 2.

\begin{tabular}{|c|c|c|c|c|c|c|c|c|c|}
\hline SNPid & $\boldsymbol{r}$ & $\boldsymbol{r}_{\boldsymbol{I}}$ & $\boldsymbol{r}_{2}$ & $\boldsymbol{r}$ & $\boldsymbol{r}_{\boldsymbol{I}}$ & $\boldsymbol{r}_{2}$ & $\boldsymbol{E}_{\boldsymbol{p}}$ & $\boldsymbol{E}_{\boldsymbol{c}}$ & $\boldsymbol{b}$ \\
\hline $\mathrm{rs} 10510126$ & 955 & 180 & 10 & 854 & 272 & 14 & 0.8085 & 0.84 & 0.7594 \\
\hline rs12505080 & 608 & 477 & 50 & 628 & 408 & 99 & 0.8976 & 0.8725 & 0.8202 \\
\hline rs17157903 & 777 & 316 & 18 & 862 & 220 & 26 & 0.8255 & 0.8453 & 0.7642 \\
\hline rs1219648 & 352 & 543 & 250 & 433 & 538 & 170 & 0.9805 & 0.9719 & 0.9585 \\
\hline rs7696175 & 353 & 605 & 187 & 396 & 496 & 249 & 0.9686 & 0.9476 & 0.9285 \\
\hline rs2420946 & 357 & 546 & 242 & 440 & 537 & 165 & 0.9792 & 0.9673 & 0.9512 \\
\hline
\end{tabular}

\section{APPENDIX}

Derivation of $\tilde{\boldsymbol{c}}_{\boldsymbol{i}}$ : From (P3), we have $c_{i}=\lim _{n} d(n) \sigma_{i, n}\left(\lambda_{0}\right) / \mu_{i, n}\left(\lambda_{0}\right)$. Also, as in the proof in Serfling (1980 [8], p. 317-318), $P_{\lambda_{0}}\left(S_{i, n}>u_{\alpha, i, n}\right) \rightarrow \alpha$ and $\beta_{i, n}\left(\lambda_{n}\right):=P_{\lambda_{n}}^{n}\left(S_{i, n} \leq u_{\alpha, i, n}\right) \rightarrow \beta$ if and only if 


$$
\frac{\left(\lambda_{n}-\lambda_{0}\right)^{k}}{k !} \frac{d(n)}{c_{i}} \rightarrow F_{i}^{-1}(1-\alpha)-F_{i}^{-1}(\beta) \text { or } \quad \frac{\left(\lambda_{n}-\lambda_{0}\right)^{k}}{k !} \frac{d(n)}{\tilde{c}_{i}} \rightarrow 1 .
$$

Thus, for $\beta_{i, n}\left(\theta_{\mathrm{n}}\right) \rightarrow \beta$, we must have;

$$
\frac{\left(\lambda_{n}-\lambda_{0}\right)^{k}}{k !} \frac{d\left(n_{1}\right)}{\tilde{c}_{1}} \sim \frac{\left(\lambda_{n}-\lambda_{0}\right)^{k}}{k !} \frac{d\left(n_{2}\right)}{\tilde{c}_{2}}, \text { or } \frac{d\left(n_{1}\right)}{d\left(n_{2}\right)} \rightarrow \frac{\tilde{c}_{1}}{\tilde{c}_{2}} .
$$

Proof of Proposition 1: We use (4) to compute $e_{p}\left(Z_{M E R T} Z\left(\theta^{(0)}\right)\right)$. By definition of $\left.Z\left(\theta^{(0)}\right)\right)$ and CLT we have $Z\left(\theta^{(0)}\right) \stackrel{D}{\rightarrow} N(0,1)$, and by Theorem 3 in ZLY, $Z_{M E R T} \stackrel{D}{\rightarrow} N(0,1)^{\circ} \quad$ Also $\left.Z\left(\theta^{(0)}\right)\right)$, and $\left.Z_{M E R T}\right)$ are jointly asymptotic normal with correlation $\left(\rho_{\theta_{i}, \theta^{(0)}}+\rho_{\theta_{j}, \theta^{(0)}}\right) / \sqrt{2\left(1+\rho_{\theta_{i}, \theta_{j}}\right)}$. Thus the condition of (4) are satisfied, and it gives;

$$
e_{P}\left(Z_{M E R T}, Z\left(\theta^{(0)}\right)\right)=\frac{\left(\rho_{\theta_{i}, \theta^{(0)}}+\rho_{\theta_{j}, \theta^{(0)}}\right)^{2}}{2\left(1+\rho_{\theta_{i}, \theta_{j}}\right)} .
$$

Proof of Proposition 2. (i) By assumption $Y \mid(\boldsymbol{x}, g) \sim N\left(\eta^{T} \boldsymbol{x}+\sum_{j=1}^{2} \log \left(\lambda_{j}\right) I_{j}(g), \sigma^{2}\right)$, where $\lambda_{1}=\lambda, \lambda_{2}=1-\theta+\theta \lambda$. As in the proof of Theorem 4 in ZLY, we have that $\sqrt{n} Z(\theta)=\sum_{i=1}^{n} V_{i}+o_{P}(1)$ where the $V_{i}=V_{i}(\theta)$ 's are i.i.d. with;

$$
\begin{gathered}
V_{i}=\sigma^{-1}\left(l_{1,1}\left(Y_{i}, \boldsymbol{x}_{i}, g_{i} \mid 1,1, \eta_{0}\right)+\theta l_{1,2}\left(Y_{i}, \boldsymbol{x}_{i}, g_{i} \mid 1,1, \eta_{0}\right)\right. \\
\left.-\left(L_{13}^{T}\left(\eta_{0}\right)+\theta L_{23}^{T}\left(\eta_{0}\right)\right) L_{33}^{-1}\left(\eta_{0}\right) l_{1,3}\left(Y_{i}, \boldsymbol{x}_{i}, g_{i} \mid 1,1, \eta_{0}\right)\right) \\
=\left(\frac{I_{1}\left(g_{i}\right)}{(1-\theta+\theta \lambda) \sigma^{2}}+\frac{I_{2}\left(g_{i}\right)}{\lambda \sigma^{2}}+\frac{\left(L_{13}^{T}\left(\eta_{0}\right)+\theta L_{23}^{T}\left(\eta_{0}\right)\right) L_{33}^{-1}\left(\eta_{0}\right) \boldsymbol{x}_{i}}{\sigma^{2}}\right) \\
\times\left(Y_{i}-\eta^{T} \boldsymbol{x}_{i}-\log (1-\theta+\theta \lambda) I_{1}\left(g_{i}\right)+\log (\lambda) I_{2}\left(g_{i}\right)\right) / \sigma \\
:=a\left(\boldsymbol{x}_{i}, g_{i}, \lambda\right)\left(Y_{i}-\eta^{T} \boldsymbol{x}_{i}-\log (1-\theta+\theta \lambda) I_{1}\left(g_{i}\right)+\log (\lambda) I_{2}\left(g_{i}\right)\right) / \sigma .
\end{gathered}
$$

Under $H_{0}: \lambda=1, V_{i} \mid\left(\boldsymbol{x}_{i}, g_{i}\right) \sim N\left(b_{0}\left(g_{i}\right), a_{0}^{2}\left(\boldsymbol{x}_{i}, G_{i}\right)\right.$, with $b_{0}\left(g_{i}\right)=0$ and $a_{0}\left(\boldsymbol{x}_{i}, g_{i}\right)=a\left(\boldsymbol{x}_{i}, g_{i}, 1\right)$. Under $H_{1}: \lambda \neq 1, \quad V_{i} \mid\left(\boldsymbol{x}_{i}, g_{i}\right) \sim N\left(b_{1}\left(g_{i}\right), a_{1}^{2}\left(\boldsymbol{x}_{i}, g_{i}, \lambda\right)\right), \quad$ with $\quad b_{1}\left(g_{i}\right)=b\left(g_{i}\right)=\log (1-\theta+\theta \lambda) I_{1}\left(g_{i}\right)+$ $\log (\lambda) I_{2}\left(g_{i}\right)$ and $a_{1}\left(\boldsymbol{x}_{i}, g_{i}\right)=a\left(\boldsymbol{x}_{i}, g_{i}, \lambda\right)$. So we have

$$
\begin{gathered}
\mu_{0}=E\left(V_{i} \mid H_{0}\right)=E\left[E\left(V_{i} \mid\left(\boldsymbol{x}_{i}, g_{i}, H_{0}\right)\right]=0, \quad\right. \text { and } \\
\mu_{1}=E\left(V_{i} \mid H_{1}\right)=E\left[E\left(V_{i} \mid\left(\boldsymbol{x}_{i}, g_{i}, H_{1}\right)\right]=E\left[\log (1-\theta+\theta \lambda) I_{1}\left(g_{i}\right)+\log (\lambda) I_{2}\left(g_{i}\right)\right] .\right.
\end{gathered}
$$

By example A in Serfling (1980 [8], p. 330), we have;

$$
\begin{gathered}
e^{-t s} M_{k}(s)=E\left[e^{-t s} E\left(e^{s V_{i}} \mid \boldsymbol{x}_{i}, g_{i}, H_{k}\right)\right]=E\left[\exp \left(\left(b_{k}\left(g_{i}\right)-t\right) s+\frac{1}{2} a_{k}^{2}\left(\boldsymbol{x}_{i}, g_{i}\right) s^{2}\right)\right], \quad(k=0,1) \\
m_{k}(t)=\inf _{s}\left[e^{-t s} M_{k}(s)\right]=E\left[\exp \left(-\frac{\left(b_{k}\left(g_{i}\right)-t\right)^{2}}{2 a_{k}^{2}\left(\boldsymbol{x}_{i}, g_{i}\right)}\right], \quad(k=0,1),\right.
\end{gathered}
$$

and

$$
\rho_{Z(\theta)}=E\left(\exp \left[-\frac{b_{1}^{2}\left(g_{i}\right)}{2\left(a_{0}\left(\boldsymbol{x}_{i}, g_{i}\right)+a_{1}\left(\boldsymbol{x}_{i}, g_{i}\right)\right)^{2}}\right]\right)=E\left(\exp \left[-\frac{b^{2}\left(g_{i}\right)}{2\left(a\left(\boldsymbol{x}_{i}, g_{i}, 1\right)+a\left(\boldsymbol{x}_{i}, g_{i}, \lambda\right)\right)^{2}}\right]\right) \text {. }
$$


Similarly, $\left.\quad \sqrt{n} Z_{M E R T}=\sum_{k=1}^{n} V_{k}\left(\theta_{i}\right)+V_{k}\left(\theta_{j}\right)\right) / \sqrt{2\left(1+\rho_{\theta_{i}, \theta_{j}}\right)}+o_{P}(1):=\sum_{k=1}^{n} V_{k}+o_{P}(1)$. We have, under $H_{0}, V_{k} \mid\left(\boldsymbol{x}_{k}, g_{k}\right) \sim N\left(b_{0}\left(g_{k}\right), a_{0}^{2}\left(\boldsymbol{x}_{k}, g_{k}\right)\right)$, with $b_{0}\left(g_{k}\right)=0$ and $a_{0}\left(\boldsymbol{x}_{k}, g_{k}\right)=$ $\left[a\left(\boldsymbol{x}_{k}, g_{k}, \theta_{i}, 1\right)+a\left(\boldsymbol{x}_{k}, g_{k}, \theta_{j}, 1\right)\right] / \sqrt{2\left(1+\rho_{\theta_{i}, \theta_{j}}\right)} ; \quad$ Under $\quad H_{1}, \quad V_{k} \mid\left(\boldsymbol{x}_{k}, g_{k}\right) \sim$ $N\left(b_{1}\left(g_{k}\right), a_{1}^{2}\left(\boldsymbol{x}_{k}, g_{k}\right)\right)$, where $\quad b_{1}\left(g_{k}\right)=b\left(g_{k}\right)=E\left(V_{k} \mid H_{1}\right)=\left[\log (1-\theta+\theta \lambda) I_{1}\left(g_{k}\right)+\right.$ $\left.\log (\lambda) I_{2}\left(g_{k}\right)\right] / \sqrt{2\left(1+\rho_{\theta_{i}, \theta_{j}}\right)}, \quad$ and $\quad a_{1}\left(\boldsymbol{x}_{k}, g_{k}\right)=\left[a\left(\boldsymbol{x}_{k}, g_{k}, \theta_{i}, \lambda\right)+a\left(\boldsymbol{x}_{k}, g_{k}, \theta_{j}, \lambda\right)\right] /$ $\sqrt{2\left(1+\rho_{\theta_{i}, \theta_{j}}\right)}$

similar to that for $\left(Z\left(\theta^{(0)}\right)\right)$.

(ii). We first compute $\tilde{Q}_{Z\left(\theta^{(0)}\right)}$. In this case, let be the weak limit of $\left(Z\left(\theta^{(0)}\right)\right)$. Then, $S \mid H_{0} \sim$ $N(0,1)$, and $S \mid H_{n} \sim N\left(\sqrt{n} \mu\left(\lambda_{n}, \theta^{(0)}\right) / \sigma\left(\theta^{(0)}\right), 1\right)$. So $\mu_{0}=E\left(S \mid H_{0}\right)=0$. Note $\mu\left(\lambda_{n}, \theta^{(0)}\right)=$ $\mu\left(\lambda_{0}, \theta\right)+\mu^{(1)}\left(\lambda_{0}, \theta^{(0)}\right) n^{-1 / 2}+O\left(n^{-1}\right)$, we have $\mu_{1}=\lim _{n} E\left(S \mid H_{n}\right)=\mu^{(1)}\left(\lambda_{0}, \theta^{(0)}\right) / \sigma\left(\theta^{(0)}\right)$. Thus

$$
\tilde{Q}_{Z\left(\theta^{(0)}\right)}=\inf _{0 \leq t \leq \mu_{1}} \tilde{Q}_{Z\left(\theta^{(0)}\right)}(t)=\inf _{0 \leq t \leq \mu_{1}}\left(1-\Phi(t)+\Phi\left(t-\mu_{1}\right)\right)
$$

Proof of Proposition 3. Since under $H_{0}, Z(\theta) \stackrel{D}{\rightarrow} N(0,1)$, we have $t_{n}(\alpha) \rightarrow \Phi^{-1} \quad(1-\alpha)$; and under $H_{1}, Z(\theta)-\sqrt{n} \mu(\lambda, \theta) / \sigma(\theta) \sim N(0,1)$. Since $\Phi(\cdot)$ is continuous on $(-\infty, \infty)$, the distribution function of $Z(\theta)-\sqrt{n} \mu(\lambda, \theta) / \sigma(\theta)$ converges to uniformly $\Phi($.$) . Note \mu(\lambda, \theta)>0$, so for $\lambda>1$ we have;

$$
\begin{gathered}
\beta_{Z(\theta), n}(\lambda)=P_{\lambda}\left(Z(\theta) \leq t_{Z(\theta), n}(\alpha)\right)=(1+o(1)) P_{\lambda}\left(Z(\theta) \leq \Phi^{-1}(1-\alpha)\right) \\
=(1+o(1)) \Phi\left(\Phi^{-1}(1-\alpha)-\sqrt{n} \frac{\mu(\lambda, \theta)}{\sigma(\theta)}\right) .
\end{gathered}
$$

Let $x=\sqrt{n}$, using L'hopital's rule twice, we get;

$$
\begin{gathered}
d_{Z(\theta)}=\lim _{n}-2 n^{-1} \log \beta_{Z(\theta), n}(\lambda) \\
=\lim _{x \rightarrow \infty}-\frac{2(1+o(1)) \log \Phi\left(\Phi^{-1}(1-\alpha)-x \frac{\mu(\lambda, \theta)}{\sigma(\theta)}\right)}{x^{2}} \\
=\lim _{x \rightarrow \infty}-\frac{2 \log \Phi\left(\Phi^{-1}(1-\alpha)-x \frac{\mu(\lambda, \theta)}{\sigma(\theta)}\right)}{x^{2}} \\
=\lim _{x \rightarrow \infty} \frac{\frac{\mu(\lambda, \theta)}{\sigma(\theta)} \phi\left(\Phi^{-1}(1-\alpha)-x \frac{\mu(\lambda, \theta)}{\sigma(\theta)}\right)}{x \Phi\left(\Phi^{-1}(1-\alpha)-x \frac{\mu(\lambda, \theta)}{\sigma(\theta)}\right)} \\
=\lim _{x \rightarrow \infty} \frac{-x\left[\frac{\mu(\lambda, \theta)}{\sigma(\theta)}\right]^{3} \phi\left(\Phi^{-1}(1-\alpha)-x \frac{\mu(\lambda, \theta)}{\sigma(\theta)}\right)}{\Phi\left(\Phi^{-1}(1-\alpha)-x \frac{\mu(\lambda, \theta)}{\sigma(\theta)}\right)-x \frac{\mu(\lambda, \theta)}{\sigma(\theta)} \phi\left(\Phi^{-1}(1-\alpha)-x \frac{\mu(\lambda, \theta)}{\sigma(\theta)}\right)} \\
=\lim _{x \rightarrow \infty} \frac{-x\left[\frac{\mu(\lambda, \theta)}{\sigma(\theta)}\right]^{3} \phi\left(\Phi^{-1}(1-\alpha)-x \frac{\mu(\lambda, \theta)}{\sigma(\theta)}\right)}{-x \frac{\mu(\lambda, \theta)}{\sigma(\theta)} \phi\left(\Phi^{-1}(1-\alpha)-x \frac{\mu(\lambda, \theta)}{\sigma(\theta)}\right)}=\frac{\mu^{2}(\lambda, \theta)}{\sigma^{2}(\theta)} .
\end{gathered}
$$


Similarly, under $\quad H_{0}, Z_{M E R T} \stackrel{D}{\rightarrow} N(0,1)$, and under $\quad H_{1}, Z_{M E R T}-\sqrt{n} \mu_{M E R T}(\lambda) \sim N(0,1) \quad$ where $\mu_{M E R T}=\left[\mu\left(\lambda, \theta_{i}\right) / \sigma\left(\theta_{i}\right)+\mu\left(\lambda, \theta_{j}\right) / \sigma\left(\theta_{j}\right)\right] / \sqrt{2\left(1+\rho_{\theta_{i}, \theta_{j}}\right)}$. The same way we get;

$$
d_{Z_{M E R T}}(\lambda)=\lim _{n} 2 n^{-1} \log \beta_{Z_{M E R T}, n}=\mu_{M E R T}^{2}(\lambda)
$$

Proof of Proposition 4. i). In our case $\Lambda_{0}=\{1\}=\left\{\lambda_{0}\right\}, \Lambda_{1}=(1, \infty)=\left(\lambda_{0}, \infty\right)$, and when $\lambda=\lambda_{0}, Z(\theta) \stackrel{D}{\rightarrow} N(0,1)$, so $L_{n}(\lambda)=\sup _{\xi \in \Lambda_{0}}\left[1-F_{n, \xi}\left(S_{n} \mid \lambda\right)\right] \sim 1-\Phi\left(S_{n} \mid \lambda\right)$ uniformly in $S_{n}$. From proof of Theorem 4 in ZLY, we have that for $\lambda \in \Lambda_{1}, n^{-1 / 2} Z(\theta) \rightarrow \mu(\lambda, \theta) / \sigma(\theta)$ (a.s.). Now we compute, for $t \in(1, \infty)$,

$$
g(t):=\lim _{n}-2 n^{-1} L_{n}=\lim _{n}-2 n^{-1}(1+o(1)) \log \left(1-\Phi\left(n^{1 / 2} t\right)\right) .
$$

Let $x=\sqrt{n}$, and use L'Hopital's rule,

$$
g(t)=\lim _{x \rightarrow \infty} \frac{-2(1+o(1)) \log (1-\Phi(x t))}{x^{2}}=\lim _{x \rightarrow \infty} \frac{-2 \log (1-\Phi(x t))}{x^{2}}=\lim _{x \rightarrow \infty} \frac{t \phi(x t)}{x(1-\Phi(x t))} .
$$

Since $\phi(x t) \rightarrow 0$, and by L'hopital's rule, $x(1-\Phi(x t))=(1-\Phi(x t)) /(1 / x)=t x^{2} \phi(x t) \rightarrow 0$, so use L'Hopital's rule on the above again,

$$
g(t)=\lim _{x \rightarrow \infty} \frac{-t^{3} x \phi(x t)}{1-\Phi(x t)-x t \phi(x t)}=t^{2} .
$$

Thus by Bahadur's (1960) [13] Theorem,

$$
c_{Z(\theta)}(\lambda)=\mu^{2}(\lambda, \theta) / \sigma^{2}(\theta)
$$

Since $Z_{M E R T}=\left(Z\left(\theta_{i}\right)+Z\left(\theta_{j}\right)\right) / \sqrt{2\left(1+\rho_{\theta_{i}, \theta_{j}}\right)}$, for $\lambda \in \Lambda_{0}, Z_{M E R T} \stackrel{D}{\rightarrow} N(0,1) ;$ for $\lambda \in \Lambda_{1}, n^{-1 / 2} Z_{M E R T} \rightarrow \mu_{M E R T}(\lambda)$ (a.s.), so $c_{Z_{M E R T}}(\lambda)$ is similarly computed.

(ii. Note $\mu\left(1, \theta_{0}\right)=0$, under $H_{0}, Z(\theta) \stackrel{D}{\rightarrow} N(0,1)$; under $H_{n}, Z(\theta) \sim \sqrt{n} \mu\left(\lambda_{n}, \theta_{0}\right) / \sigma\left(\theta_{0}\right) \sim \mu^{(1)}\left(1, \theta_{0}\right) /$ $\sigma\left(\theta_{0}\right)$ (a.s.), so

$$
e_{Z(\theta)}=\lim _{n}\left(1-\Phi\left(Z(\theta) \mid H_{n}\right)\right)=1-\Phi\left(\mu^{(1)}\left(1, \theta_{0}\right) / \sigma\left(\theta_{0}\right)\right)
$$

Similarly, under $H_{0}, Z_{M E R T} \stackrel{D}{\rightarrow} N(0,1)$; under $H_{n}, Z_{M E R T} \sim \mu_{M E R T}\left(\lambda_{n}\right) \sim \mu_{M E R T}^{(1)}(1)$, (a.s.), so

$$
\tilde{e}_{Z_{M E R T}}=\lim _{n}\left(1-\Phi\left(Z_{M E R T} \mid H_{n}\right)\right)=1-\Phi\left(\mu_{M E R T}^{(1)}(1)\right) .
$$

\section{CONSENT FOR PUBLICATION}

Not applicable.

\section{CONFLICT OF INTEREST}

The authors declare no conflict of interest, financial or otherwise.

\section{ACKNOWLEDGEMENTS}

Declared none. 


\section{REFERENCES}

[1] C.M. Sitlani, and B. McKnight, "Relative efficiency of trend tests with misspecified genetic models in stratified analyses of case-control or cohort data", Hum. Hered., vol. 71, no. 4, pp. 246-255, 2011. [http://dx.doi.org/10.1159/000328858] [PMID: 21811075]

[2] G. Zheng, "Some statistical properties of efficiency robust tests with applications to genetic association studies", Scand. J. Stat., pp. 762-774, 2014. [http://dx.doi.org/10.1111/sjos.12060]

[3] J. Gastwirth, "On robust procedures", J. Am. Stat. Assoc., pp. 929-948, 1966. [http://dx.doi.org/10.1080/01621459.1966.10482185]

[4] J. Gastwirth, "The use of maximin efficiency robust tests in combining contingency tables and survival analysis", J. Am. Stat. Assoc., pp. 380-384, 1985. [http://dx.doi.org/10.1080/01621459.1985.10478127]

[5] A. Birnbaum, "Efficiency robust 2-sample rank tests", J. Am. Stat. Assoc., pp. 1241-1251, 1967. [http://dx.doi.org/10.1080/01621459.1967.10500929]

[6] J. Rosen, The gradient projection method for non-linear programming Part I: Linear constraints., SIAM J., 1960, pp. 181-217.

[7] J. Gastwirth, "On robust rank test", In: Nonparametric Techniques in Statistical Inference, Cambridge University Press: London, 1970.

[8] R. Serfling, Approximation Theorems of mathematical Statistics Wiley., Wiley, 1980. [http://dx.doi.org/10.1002/9780470316481]

[9] Y. Nikitin, "Asymptotic relative efficiency in testing (version 5)", Stat Prob: The Encyclopedia Sponsored by Statistics and Probability Societies, 2011.

[10] Y. Nikitin, Asymptotic Efficiency of Nonparametric Tests., Cambridge University Press, 1995. [http://dx.doi.org/10.1017/CBO9780511530081]

[11] A. van der Varrt, Asymptotic Statistics., Cambridge University Press, 1998. [http://dx.doi.org/10.1017/CBO9780511802256]

[12] H. Wieand, "A condition under which the Pitman and Bahadur", Ann. Stat., pp. 1003-1011, 1976. [http://dx.doi.org/10.1214/aos/1176343600]

[13] R. Bahadur, "Stochastic comparison of tests", Ann. Math. Stat., pp. 276-295, 1960. [http://dx.doi.org/10.1214/aoms/1177705894]

[14] A. Dembo, Large deviation techniques and applications $2^{\text {nd }}$., Springer: New York, 1998. [http://dx.doi.org/10.1007/978-1-4612-5320-4]

[15] J. Deuschel, Large deviations, Academic Press: Boston, 1989.

[16] R. Bahadur, "Rates of convergence of estimates and test statistics", Ann. Math. Stat., pp. 303-324, 1967. [http://dx.doi.org/10.1214/aoms/1177698949]

[17] M. Arcones, "Bahadur efficiency of the likelihood ratio test", Math. Methods Stat., pp. 163-179, 2005.

[18] W. Hoeffding, "Asymptotically optimal tests for multinomial distributions (with discussion)", Ann. Math. Stat., pp. 369-408, 1965. [http://dx.doi.org/10.1214/aoms/1177700150]

[19] I. Sanov, On the probability of large deviations of random variables.Sel., Transl. Math. Statist. Prob, 1957, pp. 213-244.

[20] H. Rubin, "Bayes risk efficiency", Sankhya, A., pp. 325-346, 1965.

[21] W. Kallenberg, "Intermediate efficiency, theory and examples", Ann. Stat., pp. 170-182, 1983. [http://dx.doi.org/10.1214/aos/1176346067]

[22] A. Borovkov, "Large deviations and testing of statistical hypotheses", Sib. Adv. Math., 1993.

[23] E. Pitman, Lecture Notes on Nonparametric Statistical Inference, Columbia University: Mimeographed., 1949.

[24] G. Noether, "Asmptotic properties of the wald-wolfowitz test of randomness", Ann. Math. Stat., pp. 231-246, 1950. [http://dx.doi.org/10.1214/aoms/1177729841]

[25] C. Rao, "Criteria of estimation in large samples", Sankhya Ser. A, pp. 189-206, 1963.

[26] C. van Eden, "The relationship between Pitman's asymptotic relative efficiency of two tests and correlation coefficient between their test statistics", Ann. Math. Stat., pp. 1442-1451, 1963. [http://dx.doi.org/10.1214/aoms/1177703876]

[27] H. Chernoff, "A measure of asymptotic efficiency for tests of a hypothesis based on sums of observations", Ann. Math. Stat., pp. 493-507, 1952.

[http://dx.doi.org/10.1214/aoms/1177729330]

[28] W. Kallenberg, "Chernoff efficiency and deficiency", Ann. Stat., pp. 583-594, 1982. [http://dx.doi.org/10.1214/aos/1176345799] 
[29] J. Hodges, "The efficiency of some nonparametric competitors of the t-test", Ann. Math. Stat., pp. 324-335, 1956. [http://dx.doi.org/10.1214/aoms/1177728261]

[30] Y. Zang, "Simple algorithms to calculate asymptotic Simple algorithms to calculate asymptotic", J. Stat. Softw., pp. 1-24, 2010.

[31] D.J. Hunter, P. Kraft, K.B. Jacobs, D.G. Cox, M. Yeager, S.E. Hankinson, S. Wacholder, Z. Wang, R. Welch, A. Hutchinson, J. Wang, K. Yu, N. Chatterjee, N. Orr, W.C. Willett, G.A. Colditz, R.G. Ziegler, C.D. Berg, S.S. Buys, C.A. McCarty, H.S. Feigelson, E.E. Calle, M.J. Thun, R.B. Hayes, M. Tucker, D.S. Gerhard, J.F. Fraumeni Jr, R.N. Hoover, G. Thomas, and S.J. Chanock, "A genome-wide association study identifies alleles in FGFR2 associated with risk of sporadic postmenopausal breast cancer", Nat. Genet., vol. 39, no. 7, pp. 870-874, 2007. [http://dx.doi.org/10.1038/ng2075] [PMID: 17529973]

[32] Q. Li, G. Zheng, Z. Li, and K. Yu, "Efficient approximation of P-value of the maximum of correlated tests, with applications to genome-wide association studies", Ann. Hum. Genet., vol. 72, no. Pt 3, pp. 397-406, 2008. [http://dx.doi.org/10.1111/j.1469-1809.2008.00437.x] [PMID: 18318785]

(C) 2018 Yuan et al.

This is an open access article distributed under the terms of the Creative Commons Attribution 4.0 International Public License (CC-BY 4.0), a copy of which is available at: https://creativecommons.org/licenses/by/4.0/legalcode. This license permits unrestricted use, distribution, and reproduction in any medium, provided the original author and source are credited. 